\title{
Self-organization of red blood cell suspensions under confined 2D flows
}

Cécile Iss, ${ }^{a}$ Dorian Midou, ${ }^{\mathrm{b}}$ Alexis Moreau, ${ }^{a}$ Delphine Held, ${ }^{a}$ Anne Charrier, ${ }^{a}$ Simon Mendez, ${ }^{b}$ Annie Viallat*a and Emmanuèle Helfer*a

a Aix Marseille Univ, CNRS, CINAM, Marseille, France.

b Institut Montpelliérain Alexander Grothendieck, CNRS, Univ. Montpellier, Montpellier, France

*Corresponding authors : helfer@cinam.univ-mrs.fr, annie.viallat@univ-amu.fr

\begin{abstract}
Dynamic self-organized structures with long-range order have been observed in emulsions and suspensions of particles under confined flows. Here, experiments on red blood cell suspensions in quasi-2D confined flows and numerical simulations were combined to explore long-distance self-organization as a function of the channel width, the red blood cell concentration and the flow rate. They reveal and quantitatively describe the existence of red blood cell long-range alignments and heterogeneous cross-stream concentration profiles characterized by red blood cell-enriched bands parallel to the flow. Numerical simulations show that, in addition to the degree of lateral confinement, the key factor for the structural self-organization of a suspension of particles under a confined flow is the deformability of the constituent particles.
\end{abstract}




\section{Introduction}

Many-body active systems such as fish, bacteria and self-propelled particles spontaneously exhibit remarkable collective motions with ordered spatial patterns on a scale much larger than that of the constituent individuals. ${ }^{1,2,3,4}$ Under flow fields, confined emulsions and suspensions of passive microparticles also present dynamic self-organized structures with long-range order. For instance, red blood cells (RBCs) in suspension in narrow capillaries flow by irregular successions of lines in which all cells are equally spaced. ${ }^{5,6}$ Other examples are 1D microfluidic crystals formed by water-in-oil droplets at T-junction between water and oil channel, ${ }^{7}$ droplet strings and pearl necklaces morphologies displayed by immiscible twophase blends in confined shear flows, ${ }^{8,9}$ and crystal-like structures of RBCs theoretically predicted in wall-confined shear flows. ${ }^{10}$ Such patterns result from the collective dynamics of particles induced by the long-range many-body hydrodynamic interactions among them, and between them and boundary walls. Theoretical and numerical studies in recent years have described the hydrodynamic interactions between aligned particles as well as the stability of particle lines. ${ }^{11,12,13,14,15}$ However, a full description of flow structures in non-equilibrium emulsions and suspensions under confined flows is still lacking and the quantitative comparison between experimental, theoretical and numerical results remains very rare. This poor understanding of flow structures contrasts with the extended utilization of suspensions and emulsions of multiphase fluids circulating in a large variety of microfluidic- integrated devices. For example, suspensions of living cells are very commonly handled in microflows for purposes of characterization, labelling, modifications and sorting, while droplets in emulsions are massively utilized as chemical microreactors. Many of these microfluidic applications are based on quasi 2D-flows created in rectangular microchannels with a narrow depth of the order of the particle size. The advantage is that all particles flow at the same depth in the channel, i.e. at the same optical focus, thus allowing easy detection, characterization and monitoring by microscopy techniques. Yet, the behaviour of suspensions in quasi 2D confined flows, and in particular, of deformable particles such as cells and droplets, is little documented. An experimental work has reported dynamic alignments of RBCs flowing in rectangular channels of $100 \times 5 \mu \mathrm{m}^{2} .{ }^{16}$ Numerical works have explored the stability of particle alignments and have shown that in contrast with deformable particles, alignments of rigid particles are unstable when subjected to a misalignment perturbation. ${ }^{13,14}$ However, the process of cross-stream organization of the 
particles in quasi 2D confined flows, the key parameters and their role in the emergence of dynamic long-range ordered structures are poorly known.

Here, we coupled quantitative experiments and numerical simulations on RBC suspensions in quasi $2 \mathrm{D}$ confined flows to elucidate the contributions of important parameters, namely the $\mathrm{RBC}$ volume fraction in the suspension (haematocrit $\mathrm{Ht}$ ), the degree of lateral confinement (channel width), the flow rate, and the deformability of the cells, to the RBC organization in the flow. We reveal the existence of long-ordered dynamic lines of RBCs and we experimentally and numerically show that the cross-flow distribution of RBC concentration is uneven. We observe the appearance of concentrated RBCs bands at specific lateral positions, whose sharpness increases with both the lateral confinement and the particle deformability. Thanks to numerical simulations, we elucidate the underlying physical mechanisms, based on the combination of lateral confinement and cell deformability.

\section{Materials and Methods}

\section{Microfluidic chip fabrication}

The microfluidic chip is made of polydimethylsiloxane (PDMS) (Sylgard 184, Dowsil). A silicon master presenting various channel designs in positive relief $(9 \pm 1 \mu \mathrm{m}$ in height, 30 or $60 \mu \mathrm{m}$ in width and $6 \mathrm{~mm}$ in length) was fabricated in the clean room facility PLANETE (CT-PACA Micro- and Nanofabrication Platform, Marseille, France) by performing optical microlithography on SU8 resist (MicroChem) on a silicon wafer.

Before each use, a hydrophobic functionalization layer of Trichloro $(1 \mathrm{H}, 1 \mathrm{H}, 2 \mathrm{H}, 2 \mathrm{H}$ perfluorooctyl-trichlorosilane (Sigma) is deposited on the master silicon surface to prevent PDMS from sticking. The PDMS base mixed with the curing agent in a 10:1 ratio is poured on the master to form a 3-4 mm thick layer and cross-linked for $2 \mathrm{~h}$ at $65^{\circ} \mathrm{C}$. Once polymerized, the PDMS chip is carefully peeled off the master and its inlet and outlet are drilled open with a $0.75-\mathrm{mm}$ diameter punch. The microfluidic device is finally assembled by bounding the chip to a PDMS-coated coverslip after $\mathrm{O}_{2}$ plasma treatment.

\section{Microfluidic experiments}

The device is placed on an inverted microscope (IX71, Olympus) equipped with a high-speed camera (Fastcam Mini, Photron), and a $\times 20$ objective. The chip inlet and outlet are connected via Teflon tubing (0.013" inner diameter (ID) and $0.027^{\prime \prime}$ outer diameter (OD) for the inlet, $0.015^{\prime \prime} I D$ and 0.027 " OD for the outlet) to a flow controller system (MFCS-8C, 
Fluigent) that allows controlling the pressure drop between inlet and outlet and the circulation of solutions in the microfluidic channel.

Before injection of the sample, the channel is passivated using DPBS pH 7.4 (Dulbecco's Phosphate Buffer Saline without Calcium and Magnesium, Gibco) adjusted with glucose to 295 mOsm (named DPBS-G) and supplemented with 0.5\% BSA (Bovine Serum Albumine, Sigma). After 30-min incubation the channel is rinsed with DPBS-G and is ready for use. The RBC suspension at $10 \% \mathrm{Ht}$ is injected in the channel via a pipet tip directly plugged to the inlet via Teflon tubing (Fig. 1A, top). Movies of RBCs flowing through the field of view (typically 500-600 $\mu \mathrm{m}$ long) are acquired in bright field at a high frame rate (1600 to 2000 $\mathrm{fps})$. The videos are acquired either at the entry of the channel or $6 \mathrm{~mm}$ further away at the exit. All experiments are performed at room temperature. To limit RBC sedimentation and prevent subsequent channel obstruction, the pipet tip containing the sample is kept vertical and the inlet tubing is as short and vertical as possible. In this configuration $\mathrm{Ht}$ increases slowly enough during experiment so that it can be considered constant during the acquisition of a movie (a few seconds long). In addition, the sample is replaced every 5 to 10 min depending on its concentration and the applied pressure drops.

\section{Video analysis}

The images are processed with ImageJ to track either individual RBCs (in single images) or $\mathrm{RBC}$ linear clusters (in movies). The average $\mathrm{Ht}$ is calculated as the ratio of the volume of the total number of tracked RBCs $\left(90 \mu \mathrm{m}^{3}\right.$ per RBC) and the volume represented by the analysed images.

Single RBC tracking. Individual RBCs are tracked automatically for $\mathrm{Ht}$ below $5 \%$ as they are distinct: the image is first binarized (RBCs and background are in black, RBCs being separated from background by a white contour), then the background is removed (only black RBCs left with a white background), and RBC positions $(x, y)$ are determined using Ellipse Split ImageJ plugin. Above 5\%, due to crowding, RBCs cannot be automatically discriminated as single objects and they are tracked manually: the position of their centre of mass is selected by eye (Supp. Fig. S3, left). At $\mathrm{Ht}<5 \%$ all the RBCs observed in a movie are tracked to count as many cells as possible (typically 50 to 150) whereas for higher $\mathrm{Ht}$, two images of the movie are enough to count at least 200 cells. From the individual positions are plotted the RBC crossflow distribution through the channel width (Supp. Fig. S3, right) and the position dot plot (Supp. Fig. S3, middle) using Igor software (WaveMetrics Inc.). In the dot plot each dot 
corresponds to one tracked RBC: the dots are positioned at the exact y-position (y-axis) and randomly spread on the $\mathrm{x}$-axis; the overlapping between the dots is adjusted to compromise between clarity of the distribution and limited plot spreading, the same overlapping parameter is used for simultaneously displayed plots (Supp. Fig. S4).

$R B C$ train tracking. For linear clusters (named 'trains' in the text), the position (centre of mass), length (end-to-end) and number of RBCs are measured when the train first appears in the field of view. Clusters are considered as trains when moving with the same cells through the field of view for more than $240 \mu \mathrm{m}$. Once they have moved this distance, any change in cell number defines a new train (in case of cell addition) or two new trains (in case of dissociation into two parts) in case they further move over $240 \mu \mathrm{m}$.

Experiments performed at the same pressure drop with close $\mathrm{Ht}$ (less than $2 \%$ variation) were pooled to increase the statistics and to reduce noise.

\section{Blood sample preparation}

Blood samples are harvested from voluntary healthy donors $(\approx 30 \mu \mathrm{L}$ via pinprick) and immediately processed. RBCs are extracted by washing the blood sample 3 times in DPBS-G (3-min centrifugation at $500 \mathrm{rcf}$ at RT) and finally resuspended at $10 \% \mathrm{Ht}$. $30 \mu \mathrm{L}$ of this suspension are immediately pipetted using a $200-\mu l$ pipet. The tip with the RBC suspension is taken off the pipet and directly connected to the inlet (Fig. 1A, top) while its top is connected to the MFCS tubing via an adaptor.

Cell rigidification. RBCs freshly harvested are diluted to $0.2 \% \mathrm{Ht}$ in DPBS-G supplemented with $0.1 \%$ glutaraldehyde (Sigma) and incubated for at least $15 \mathrm{~min}$ at $4^{\circ} \mathrm{C}$. RBCs are then washed 3 times in DPBS-G before final resuspension at $10 \% \mathrm{Ht}$ for immediate use.

\section{Results}

\section{Observations}

Figure $1 \mathrm{~A}$ depicts the flow experiment: The RBC suspension is injected in a rectangular crosssection channel via the inlet, under a controlled pressure drop (20, 35 and 50 mbar yield velocities of RBCs around 0.8-1, 1.5-2 and 3-4 mm/s, respectively). RBCs flowing in the $x-$ direction are observed in the channel using bright field microscopy, either close to the entry ( $\left.\mathrm{L}_{\text {in }}\right)$ or just before the exit ( $\left.\mathrm{L}_{\text {out }}\right)$, at the end of the 6-mm channel length) (Fig. $1 \mathrm{~A}$, bottom). Figure 2 and Supp. Movies M1-M2 display typical observations of flowing RBCs at $L_{\text {in }} / L_{\text {out }}$ positions, in channels of 30- or 60- $\mu \mathrm{m}$ widths (ML30 and ML60, respectively), and at various 
$\mathrm{Ht}$, under a pressure drop of 20 mbar. Figure $2 \mathrm{~A}$ compares the structure of the flow at $\mathrm{L}_{\text {in }}$ and Lout in an ML60 channel at increasing Ht (Supp. Movie M1 displays flows at 15\% Ht), while Fig. 2B shows the flow at Lout for ML60 and ML30 channels at $12.5 \% \mathrm{Ht}$ (see corresponding Supp. Movie M2).

RBC shapes, vertical position and velocities. Healthy RBCs deform under the effect of friction imposed by the top and bottom walls and adopt a specific shape, close to slipper or parachute-shapes, as observed in several previous studies. Lift forces ${ }^{17,18,19,20}$ cause the RBCs to migrate away from the top and bottom walls towards regions of lower shear rate at mid-z height, thus resulting in a $2 \mathrm{D}$-flow in a unique $\mathrm{x}$-y plane. $\mathrm{RBC}$ velocity along the flow direction was experimentally measured at $L_{\text {in }}$ and $L_{\text {out }}$ positions of an ML60 channel and the corresponding profiles at mid-z height, normalized by the maximal velocity of RBCs at the centre of the channel, are shown in Fig. 1B. These profiles are consistent with the one computed using $\mathrm{COMSOL}^{\circledR}$ software for a Newtonian fluid in a channel of $10 \times 60 \mu \mathrm{m}^{2}$ rectangular cross-section after normalization by the maximal fluid velocity at the centre of the channel (Fig. 1B). It clearly appears that the velocities of the suspending fluid and of RBCs at both channel entrance and exit are almost independent of the lateral y-position and only decrease significantly at a distance less than $5 \mu \mathrm{m}$ from the lateral walls.

RBC lateral positions. Above $10 \% \mathrm{Ht}$, RBCs spread all over the channel width with no apparent self-organized structures at $\mathrm{L}_{\text {in. }}$. In contrast, $6-\mathrm{mm}$ downstream of the inlet, at $\mathrm{L}_{\text {out, }}$ the RBC flow presents three striking features: first, RBCs are away from the channel walls, leaving a plasma skimming layer free of RBCs near the wall, called the cell-free layer; ${ }^{21}$ second, dynamic self-organized RBC clusters are observed, mostly linear and oriented along the flow; third, for a specific range of $\mathrm{Ht}$, these lines tend to stack parallel to each other over the width of the channel, especially in the narrowest channel of $30-\mu \mathrm{m}$ width (Fig. 2B), generating alternating bands of high and low RBC concentration along the y-direction. This latest feature will be further discussed in the following.

Suspensions of rigid RBCs. The flow behaviour at Lout of RBCs rigidified with glutaraldehyde is shown in Fig. 3 and in Supp. Movie M3. In strong contrast with deformable RBCs, stiffened RBCs do not migrate away from the walls: they circulate at different heights in the channel and many of them marginate towards a wall and flow at a much lower velocity than those located in the centre of the channel. Moreover, as RBCs remain discoid after rigidification they present a non-stationary tumbling motion, which prevents cell stacking and formation 
of aligned clusters. Indeed, no self-organization of the suspension is observed. The observation of RBC suspensions containing $85 \%$ of deformable RBCS and $15 \%$ of rigidified ones (Supp. Movie M4) clearly shows that the RBCs flowing close to the walls are the rigidified ones, whereas none of them are found in aligned clusters.

\section{Red blood cells cluster into trains}

As clearly shown in Figs. 2 and 4, RBCs self-organize in the direction of flow by stacking quite closely and thus form linear clusters of different lengths. These alignments are dynamic structures, they can form by merging two smaller lines or by adding a single RBC to a line (Fig. 4B) and can break along the flow, for example after a collision (Fig. 4C).

The most stable and linear structures, called trains, are defined as follows: 1) a train contains at least 3 RBCs; 2) the spacing between two consecutive RBCs of the same train is equal to or less than $1 \mu \mathrm{m} ; 3$ ) a train may be curved but its local curvature (the angle formed by the two segments connecting an RBC to its nearest neighbour, respectively at its front and rear) is less than $40^{\circ}$; 4) a train must be stable, i.e. its RBC content is constant while moving over a distance of at least $240 \mu \mathrm{m}$. We observe that these trains steadily move along the flow. At mid $\mathrm{Ht}(\approx 7-10 \%)$, train velocities are similar to that of isolated RBCs, or slightly slower (up to $10 \%)$, whatever the train length. In such dense suspensions, all objects, whether trains or single RBCs, are affected by their surroundings and vice-versa and thus cannot move independently, leading to a quasi uniform velocity.

Train-structures develop along the 2D-flow. At $L_{\text {in }}$, only a few percent of flowing RBCs are embedded in a few trains. At Lout, the amount of RBCs in trains increases as well as the number of trains. Furthermore, trains are longer in average. This is illustrated in Fig. 4D: in this example, the number of trains increases from 12 to 23 between $L_{\text {in }}$ and $L_{\text {out, }}$ their average length increases from 3.6 to $4.5 \mu \mathrm{m}$, and the percentage of RBCs in trains notably increases from $6 \%$ to $17 \%$.

Distribution of the number of RBCs per train. Typical distribution functions of the number of RBCs per train are shown for ML30 and ML60 channels, at various $\mathrm{Ht}$ and pressure drops in Figs. 5A-B. At a given pressure drop, the distribution widens at increasing $\mathrm{Ht}$ (Fig. 5A), showing that longer trains are formed when the probability of RBC encounters increases, as seen in ML30 channels at 20 and 35 mbar and in ML60 channels at 20 mbar (Fig. 5C). However, in the widest ML60 channels, at high $\mathrm{Ht}(\approx 13-15 \%)$, increasing the pressure drop 
surprinsingly shifts the distribution down to shorter values thus leading to smaller average train length (Figs 5B and 5D). In this case, the dynamical effets are actually enhanced. Many RBC linear clusters break, merge or receive additional cells over a short distance. Though it is not completely clear why an increase in capillary number results in a decrease of the number of the longest trains, one has to keep in mind that the relative velocity between interacting cells increases with the capillary number. ${ }^{14}$ Consequently, over the reference distance along which a RBC cluster must keep its cell content to be defined as a train, the probability to be perturbed by neighbouring cells increases with the hydrodynamic stress. This effect is enhanced for long trains where neighbouring cells are more numerous, which may lead to a decrease of the number of long clusters stable enough to be considered as trains. Interestingly, the values of the standard deviation plotted versus the values of the average length of each studied distribution (for the different channel widths, pressure drops and $\mathrm{Ht}$ ) describe a single master straight line. Similarly, the kurtosis plotted versus the skewness of all the studied distributions fall on a single master curve (Supp Fig. S1). The consistence between the various distributions, whatever the varied parameters, comfort the choice of the criteria used for train definition.

RBC trains are densely packed. The observed trains are different from the 3D-rouleaux observed in blood in absence of flow, which depend on the presence of fibrinogen and globulin in the plasma. ${ }^{22}$ They also seem to be much denser than the linear RBC clusters observed in cylindrical microcapillaries, where most neighboring cells are not in direct contact, even though the cell inter-distance was shown to decrease with increasing flow5. Here, RBCs almost touch each other, and the packing seems independent of the concentration and the applied pressure drop (Supp. Fig. S2). Probably due to the same mechanisms of formation, alike trains are visible in channels similar to ours but with a width of $100 \mu \mathrm{m} .{ }^{16}$ The associated physical mechanisms will be discussed when describing the results of numerical simulations.

\section{Cross-flow distribution of RBC haematocrit}

The cross-flow distribution of RBCs (the distribution normal to the flow direction) is first illustrated by displaying cell positions in dot plots at various $\mathrm{Ht}$ for ML30 and ML60 channels under a pressure drop of 20 mbar (Fig. 6 and Supp Figs. S3-S4). As mentioned earlier, the $\mathrm{RBC}$ cross-flow distribution initially uniform at $\mathrm{L}_{\text {in }}$ becomes non-uniform at Lout (Supp. Fig. S4) 
with a transverse distribution which depends on $\mathrm{Ht}$. As clearly seen in the ML30 channel a wide cell free layer and a quasi-homogeneous local RBC distribution all over the channel width are observed at low $\mathrm{Ht}(<10 \%)$, whereas two bands with high local RBC concentration appear at medium $\mathrm{Ht}$ range (10-12\%), and three bands at higher $\mathrm{Ht}(18 \%)$. In the ML60 channel, bands are observed at $14 \% \mathrm{Ht}$, which are not detected at lower $\mathrm{Ht}$ (5\%). Notably, these bands vanish at higher $\mathrm{Ht}(17 \%)$ and $\mathrm{RBCs}$ arrange in a dense and disordered packing. The concentration profiles of RBCs along the y-direction (local Ht) in ML30 and ML60 channels at different pressure drops and at different global $\mathrm{Ht}$ are shown in Fig. 7. The progressive structuration in bands is clearly seen in the ML30 channel at both 20 and 35 mbar (Figs. 7A-B). The two-band pattern is shifted towards a higher $\mathrm{Ht}$ at $35 \mathrm{mbar}$. A similar pattern is observed in the ML60 channel but with four bands, which are though less pronounced (Figs. 7D-F). Under 20 mbar pressure drop the four bands are equidistant at $11.5 \% \mathrm{Ht}$ whereas they are shifted toward the walls at $14.5 \% \mathrm{Ht}$ with an increased local $\mathrm{Ht}$ in the centre. For $\mathrm{Ht}$ below $11.5 \%$ and above $14.5 \%$ the local RBC concentration profile is homogeneous over the channel width except close to the wall, where it dramatically drops. This region is wide and devoid of RBCs at low $\mathrm{Ht}$, as expected from the cell free layer. At high $\mathrm{Ht}$ however, the deprived region is very narrow and still contains RBCs due to high crowding of the suspension. At 35 mbar, four bands are also observed at $10.4 \%$ but vanish at $12.4 \%$, at a concentration lower than that observed at 20 mbar. Interestingly, the band pattern is equivalent at 20 mbar and $35 \mathrm{mbar}$ for $\mathrm{Ht}$ values of $11.5 \%$ and $10.4 \%$, respectively.

\section{Numerical simulations}

In order to understand the physical origin of the uneven cross-flow RBC profile, we performed 3D numerical simulations in a $30-\mu \mathrm{m}$ wide channel under a prescribed pressure loss corresponding to an experimental pressure drop of $20 \mathrm{mbar}$, and for various $\mathrm{Ht}$ ranging from 2 to 20\%. The computational domain is a parallelepiped $200 \mu \mathrm{m}$ long in the flow direction, $30 \mu \mathrm{m}$ wide and $8 \mu \mathrm{m}$ high. In the flow direction, periodic boundary conditions are imposed so that the flow is fully developed and the evolution in space (along the channel path) in the experiment is mimicked by an evolution in time in the simulations. A source term is applied in the flow direction, corresponding to a pressure gradient of $200 \mathrm{~Pa} / \mathrm{mm}$, yielding typical RBC velocities of $0.8-1 \mathrm{~mm} / \mathrm{s}$. This is comparable to the $20-\mathrm{mbar}$ pressure drop ( $\approx 300 \mathrm{~Pa} / \mathrm{mm}$ between the channel extremities), which indeed yields experimental 
velocities of the order of $1 \mathrm{~mm} / \mathrm{s}$. Wall boundary conditions are imposed in the z-direction. In the $y$-direction we use both wall boundary conditions to reproduce the $30-\mu \mathrm{m}$ wide channel configuration, or periodic boundary condition to remove the effect of lateral walls. The domain is initially filled with particles (RBCs or spherical capsules) which are let evolve under flow, for a time corresponding to a travel of approximately $2 \mathrm{~mm}$ in the flow direction. $\mathrm{Ht}$ profiles in the $\mathrm{y}$-direction (Fig. $7 \mathrm{C}$ ) are obtained by integrating 3D maps of local RBC presence in the $\mathrm{x}$ - and $\mathrm{z}$-directions on the final solution of the calculation.

Method and RBC/capsule modelling. Numerical simulations are performed using an inhouse solver named YALES2BIO ${ }^{\ddagger}$ dedicated to the simulation of blood flows. Navier-Stokes equations are solved with a high-order finite-volume solver 23,24 and the effect of RBCS on the flow is accounted for using an immersed boundary method. ${ }^{25,26}$ The detailed methodology, extensive validations and recent applications of the flow solver to RBC dynamics have been published in the recent years. ${ }^{25,26,27,28,29,30,31}$ Two different series of calculations were performed using either RBC or capsule suspensions, whose characteristics are described below. The typical resolution of the fluid grid and particles meshes (RBCs and capsules) is 0.3 $\mu \mathrm{m}$.

RBC suspensions. Simulations of RBC suspensions at different $\mathrm{Ht}$ are reported. All RBCs are identical: they are modelled as a drop of viscous liquid enclosed in a thin membrane discretized with triangular elements. The ratio between the internal viscosity and the external viscosity (aqueous solution) is set to $\lambda=5$. The RBC membrane resists shear, area changes and bending. The in-plane elastic resistances are modelled by the classical Skalak hyper-elastic law ${ }^{32}$ (with high values of area modulus to prevent area changes) while bending resistance is derived from the Helfrich energy. ${ }^{33,34}$ The shear modulus is set to $G_{s}=$ $2.5 \mu \mathrm{N} / \mathrm{m}$, which is a classical value when using the Skalak model ${ }^{30,31,35,36}$, the bending modulus is set to $E_{b}=310^{-19} \mathrm{~J}$, and the spontaneous curvature is set to $C_{0}=0$, the same values used in recent simulations in shear flow. ${ }^{30-31}$ For the in-plane elastic resistance, a stress-free shape needs to be prescribed. Recent simulations have demonstrated that the stress-free shape of an RBC is not the discoid one but an oblate spheroid close to a sphere. ${ }^{37,38,39,40}$ In a preliminary simulation using the same mechanical parameters as in the flow simulations, an oblate spheroid of reduced volume 0.96 with a surface area equal to that of the RBC (133.4 $\left.\mu \mathrm{m}^{2}\right)$ is first deflated to the RBC volume $\left(92.8 \mu \mathrm{m}^{3}\right)$ until the equilibrium is reached. Copies of 
this prestressed $\mathrm{RBC}$ are then randomly positioned in the computational domain at the desired concentration.

Capsule suspensions. Three calculations of capsule suspensions are used to investigate the role of particle deformability. In order to avoid the flipping dynamics of rigidified RBCs (discoids in the experiments) which are difficult to analyse, spherical capsules of $6.5 \mu \mathrm{m}$ in diameter are considered. The same laws are used to describe membrane mechanics, except that the area modulus is now equal to the shear modulus. For the three simulations (C1-C3), the elastic properties of the capsules are modified to increase their deformability from C1 to C3 (see set values of $\lambda, G_{s}$ and $E_{b}$ in Table 1).

Numerical cross-stream RBC concentration profile. Supplemental Movie M5 shows the simulation of the temporal evolution of an RBC flow at $12 \% \mathrm{Ht}$ in a $30-\mu \mathrm{m}$ wide channel. It represents the progressive flow structuration while the cells move along the channel, the numerical time being equivalent to a distance experimentally travelled in the channel. As experimentally observed, a dynamic formation of trains is observed and the cross-flow distribution of RBCs progressively becomes non-uniform. Typical images of long-time flows in the range of experimentally used $\mathrm{Ht}$ are shown in Fig. 8. The similarity of images between in silico and real experiments is striking. Not only RBC shapes look the same but train morphologies obtained in silico are very close to experimental ones. Bands are also observed in silico, the number and positions of which are very similar to experimental ones. Simulations of RBC concentration profiles in a 30- $\mu \mathrm{m}$ wide channel for $\mathrm{Ht}$ ranging between $4 \%$ and $20 \%$ are in very good quantitative agreement with experiments (Figs. 7A and 7C). Numerical profiles are sharper than the experimental ones, which may be explained by the presence of $\mathrm{Ht}$ fluctuations in the experiment, which is not the case in simulations.

When examining experimental and numerical images, two physical quantities seem to strongly contribute to the self-organization of flow in these channels: First, the deformability of the particles which, as shown in Refs. 13 and 14, stabilizes alignments of deformable particles, whereas lines of rigid particles are unstable; Second, the degree of confinement induced by the lateral walls, whose effect is to compress particle alignments in parallel bands along the flow direction. We therefore numerically investigated the role of lateral walls and the role of particle stiffness on the flow structure.

Role of confinement. The role of the lateral walls was investigated at $4 \%$ and $12 \% \mathrm{Ht}$, in a 30 $\mu \mathrm{m}$ wide channel and in a channel with periodic conditions in the $y$-direction (non-confined 
situation) (Fig. 9). In the non-confined situation, cross-flow heterogeneity is not obvious, or is visible only locally. Longer simulations (simulation time almost doubled, not shown) did not show a tendency to form a more global structure. At $12 \% \mathrm{Ht}$, though, train-like structures are observed whose stability was however not assessed. In contrast, under lateral confinement, one band in the centre of the channel is observed at $4 \% \mathrm{Ht}$ and two parallel bands are observed at $12 \% \mathrm{Ht}$ with well-aligned structures and long train-like structures. This shows that when $\mathrm{Ht}$ is high enough for the RBCs to hydrodynamically interact with each other and to have a high probability to encounter, clusters can be formed even in nonconfined conditions. This is consistent with the previous experimental observation of trains in 100- $\mu \mathrm{m}$ wide channels by Abkarian et al. ${ }^{16}$ In presence of walls, the lateral confinement strongly favours RBC alignment, probably because the existence of a cell free layer further confines the RBCs in the central region of the channel, thus increasing the local RBC concentration and favouring encounters. Furthermore, lateral confinement clearly promotes the formation of bands parallel to the lateral walls.

Role of deformability. The flow structure of spherical capsules of $6.5 \mu \mathrm{m}$ in diameter with three different sets of mechanical properties (viscosity, shear and bending moduli; see C1-C3 in Table 1) was investigated in a 30- $\mathrm{mm}$ wide channel under the same pressure gradient as for RBC simulations. The volume fraction is set to $18 \%$, which yields a number of particles per unit length of channel similar to the $12 \% \mathrm{Ht}$ case for RBCs. The initial capsule distribution is the same for the three cases. The self-organization of the capsule flows is displayed in Fig. 10 at five evenly spaced successive times (from $t_{0}$ to $t_{4}$ ). The flow of the suspensions of the two most deformable capsules ( $\mathrm{C} 2$ and $\mathrm{C} 3$ ) becomes more and more organized over time. The suspension of the most deformable capsules (C3) ends up with two very ordered bands at the longest time while the two bands of the other suspension (C2) display a weaker order. The less deformable suspension (C1) shows some degree of self-organization, but extremely slow. For instance, the structure of $C_{1}$ suspension at $t_{4}$, quite close to that at $t_{3}$, is very similar to the structure of $\mathrm{C} 3$ suspension at time $t_{1}$. A very long calculation would be needed to assess if the $\mathrm{C} 1$ suspension can indeed self-organise.

\section{Discussion and conclusion}

In $30-$ and $60-\mu \mathrm{m}$ wide channels, we experimentally showed the progressive formation of dynamic compact RBC alignments along the flow that are stable when travelling over 
hundreds of microns. Such trains have also been observed in a $100-\mu \mathrm{m}$ wide channel. ${ }^{16} \mathrm{It}$ is important to note that no macromolecules which could induce intercellular adhesion ${ }^{5,22}$ are present in the suspension. Accordingly, the numerical simulations of deformable particles performed in the present study predicted that the formation of aligned structures resulted only from hydrodynamic interactions. Simulations clearly showed that the formation of such files is due to the deformability of the particles. This is in line with our experimental observations showing that rigidified RBCs do not contribute to train structures. Refs. 13 and 14 provide some fundamental basis to understand train formation: when confined between two parallel plates, a deformable particle generates a quadrupolar perturbation of the flow which in turn generates a relative motion between neighbouring particles, yielding 1) a preferred inter-particle distance in a pair, and 2) an alignment of particles. This mechanism explains the formation of trains even without lateral wall confinement. However, this process needs particles to be close to each other.

The lateral confinement induces another phenomenon also associated with particle deformability: the migration of the RBCs away from the wall. The associated lift force is sufficient to make an isolated RBC go to the centre $(y=0)$ of a $30-\mu$ m wide channel (additional simulation, not shown). This is not the case in a $60-\mu \mathrm{m}$ wide channel although a large cell free layer is observed at low $\mathrm{Ht}$ (Fig. 2A, top). In the latter case, the lift force due to the lateral walls tends to concentrate the cells in the bulk of the channel, but it is not high enough to push them at the centre, and the aligned structures experimentally observed in ML60 channels are not very different from that of the unconfined case predicted by simulations (see Figs. 2 and 9). However, strong lateral confinement in the $30 \mu \mathrm{m}$-wide channel has a tremendous impact on file formation and stability. Regular and stable bands are easily formed as the effect of lateral wall is felt over the whole channel width.

We both experimentally and numerically showed that the files laterally stack together. It results in a non-homogenous cross-flow concentration profile, with bands highly concentrated in RBCs along the flow, particularly marked in the case of strong confinement, and whose number is determined by the RBC concentration. Numerical simulations showed that efficient band formation requires both lateral confinement and deformable particles. It is interesting to note that the formation of bands is a local process. In particular, experiments and simulations in the 30- $\mu \mathrm{m}$ wide channel show that for some values of $\mathrm{Ht}$ (typically 6 to $9 \%$ ), the number of RBCs per unit channel length is too high to have all RBCs in 
an unique band at the centre of the channel. As a consequence, the suspension of RBCs is structured in alternate regions of one and two bands (Fig. 8 and Supp. Movie M6).

In conclusion, a key factor for the structural self-organization of a suspension of particles under confined flow is the deformability of the constituent particles, which is essential both for the local organization in trains and the migration away from the lateral walls. In the 10$20 \%$ concentration range, the existence of peaks in the particle concentration profile, corresponding to bands in the channels, depends on the deformability of the particles. The characterization of these peaks that can be easily determined by analysing an image of the flow obtained in a very simple microfluidic circuit could pave the way for a method to assess the deformability of RBCs in health and disease, and of various microscopic particles.

\section{Conflicts of interest}

There are no conflicts to declare.

\section{Acknowledgements}

This work has been carried out thanks to the support of the A*MIDEX project (n ANR-11IDEX-0001-02) and the RheoBlood project (Labex Numev ANR-10-LABX-20), both funded by the Investissements d'Avenir French Government program, managed by the French National Research Agency (ANR). S.M. thanks Dr. Moureau and Dr. Lartigue (CORIA, UMR 6614) and the SUCCESS scientific group for providing the YALES2 code, which served as a basis for the development of YALES2BIO. Simulations with YALES2BIO were performed using HPC resources from GENCI-CINES (Grants No. 2017-A0020307194 and A0040307194) and from the platform MESO@LR. 


\section{Tables}

\begin{tabular}{|c|c|c|c|}
\hline & $\begin{array}{c}\text { Internal-to-external } \\
\text { viscosity ratio } \lambda\end{array}$ & $\begin{array}{c}\text { Shear Modulus } \\
\mathrm{G}_{\mathrm{s}}(\mathrm{N} / \mathrm{m})\end{array}$ & $\begin{array}{c}\text { Bending } \\
\text { modulus } \mathrm{E}_{\mathrm{b}}(\mathrm{J})\end{array}$ \\
\hline Case C1 & 10 & $210^{-5}$ & $2.410^{-18}$ \\
\hline Case C2 & 5 & $110^{-5}$ & $1.210^{-18}$ \\
\hline Case C3 & 2 & $410^{-6}$ & $4.810^{-19}$ \\
\hline
\end{tabular}

Table 1. Set values of mechanical properties of the capsules used to assess the effect of deformability in the flow structuration. 


\section{Figure captions}

Figure 1. Red blood cells in confined 2D-flow.

A) Schematics of the flow experiment. Top, microfluidic chip: Inlet and outlet are connected to MFCS (closed circuit) for application of a controlled pressure drop $(\Delta \mathrm{P})$ to control the flow velocity inside the channel. A pipet tip containing the RBC sample is connected to the inlet through a short flexible Teflon tubing. Bottom, microfluidic channel: The flow channel is 6 $\mathrm{mm}$ long (x-direction), a few tens of $\mu \mathrm{m}$ wide (y-direction) and $9 \pm 1 \mu \mathrm{m}$ high (z-direction) so that RBCs move within a single horizontal $x$-y layer. B) Velocity profiles along the $y$-direction in a $60-\mu \mathrm{m}$ wide channel, normalized by the maximal velocity at the channel centre $(y=0)$, plotted over the half-channel $(y=[0 ; 30] \mu \mathrm{m})$ : $\mathrm{COMSOL}^{\circledR}$ simulation of the flow velocity profile (in black) and RBC velocities (averaged over 3 to 4 cells randomly selected in the field of view at same y-position), at the entry and the exit of the channel (in blue and red, respectively). $\Delta \mathrm{P}=20 \mathrm{mbar}$.

Figure 2. Red blood cell flow structuration along the 2D-channel.

A) Flows of RBCs at entry ( $L_{\text {in }}$, left) and exit ( $L_{\text {out }}$, right) of $60-\mu \mathrm{m}$ wide channels (ML60) at increasing $\mathrm{Ht}$. B) Flow structuration at $\mathrm{L}_{\text {out }}$ in 60 - and 30- $\mu \mathrm{m}$ wide channels (ML60 and ML30, respectively) at $12.5 \% \mathrm{Ht} . \Delta \mathrm{P}=20 \mathrm{mbar}$. Scale bars: $20 \mu \mathrm{m}$.

Figure 3. Rigid red blood cells do not organise under flow.

Flow image of stiffened RBCs at $13.5 \% \mathrm{Ht}$ in an ML60 channel. $\triangle \mathrm{P}=20$ mbar. Scale bar: 20 $\mu \mathrm{m}$.

Figure 4. Trains of red blood cells.

A-C) Images of trains (highlighted in red) in the RBC flow. Trains of various lengths (A) encounter dynamic events in their lifetime: they can increase in length due to addition of single RBCs or fusion with another train (B), or break down due to dissociation induced by collision with an obstacle, e.g. a white blood cell (C). ML60 channels, $\Delta \mathrm{P}=20$ mbar. Scale bars: $20 \mu \mathrm{m}$. D) Distribution of train length (in number of RBCs) at entry ( $L_{\text {in }}$ ) and exit ( $\left.L_{\text {out }}\right)$ of an ML60 channel at $\mathrm{Ht} \approx 7 \%$, under a 35 -mbar pressure drop. $\mathrm{N}$ : number or trains. Total number of RBCs (in trains and isolated): $\approx 650$.

Figure 5. RBC train formation depends on $\mathrm{Ht}$ and pressure drop.

A) Effect of $\mathrm{Ht}$ at 20- and 35-mbar pressure drops in ML30 channels. B) Effect of pressure drop at $\mathrm{Ht} \approx 13-15 \%$ in ML60 channels. $\mathrm{N}$ : number or trains. C-D) Average train length as function of $\mathrm{Ht}$, at various channel width and pressure drop (C), and as function of pressure drop in ML60 channel at $\mathrm{Ht} \approx 13-15 \%$ (D). The dashed line in $(C)$ is a guide to the eye.

Figure 6. Red blood cell cross-flow distribution.

Dot plots and typical images of RBC flow at increasing Ht in ML60 (A) and ML30 (B) channels. Scale bars: $20 \mu \mathrm{m}$.

Figure 7. Experimental and simulated cross-flow distributions of RBCs over the channel width.

A-B) RBC distributions measured in ML30 channels at various $\mathrm{Ht}$, under 20- and 35-mbar pressure drops. C) Simulation of RBC distribution in a $30-\mu \mathrm{m}$ wide channel for $\mathrm{Ht}$ ranging 
from 4 to $20 \%$, under a pressure gradient of $200 \mathrm{~Pa} / \mathrm{mm}$. D-E) RBC distributions measured in ML60 channels at various $\mathrm{Ht}$, under 20- and 35-mbar pressure drops. $\mathrm{F}$ ) Comparison of RBC distribution at $\approx 11 \% \mathrm{Ht}$ between the two pressure drops. All half-channel distributions have been symmetrized for clarity.

\section{Figure 8. Simulations of RBC flow.}

Left: Images of long-time RBC flows in a $30-\mu \mathrm{m}$ wide channel at increasing $\mathrm{Ht}$. The solid and dashed lines denote wall and periodic boundary conditions, respectively. Right: Typical images from experimental movies in ML30 channels at various $\mathrm{Ht}$ in a range similar to the one used in the simulations.

\section{Figure 9. Effect of lateral confinement.}

Simulations of RBC flows at $4 \%$ and $12 \% \mathrm{Ht}$ in absence or in presence of walls ( $30-\mu \mathrm{m}$ apart). Solid and dashed lines denote wall and periodic boundary conditions, respectively.

\section{Figure 10. Role of deformability.}

Simulations of the structuration in time of three suspensions of capsules with increasing deformability from C1 to C3 (see mechanical parameters in Table 1) in a 30- $\mu \mathrm{m}$ wide channel. Capsules are $6.5-\mu \mathrm{m}$ diameter spheres, at a volume fraction of $18 \%$. Simulation results are displayed for 5 evenly spaced instants, with an equal initial distribution for the three suspensions. 

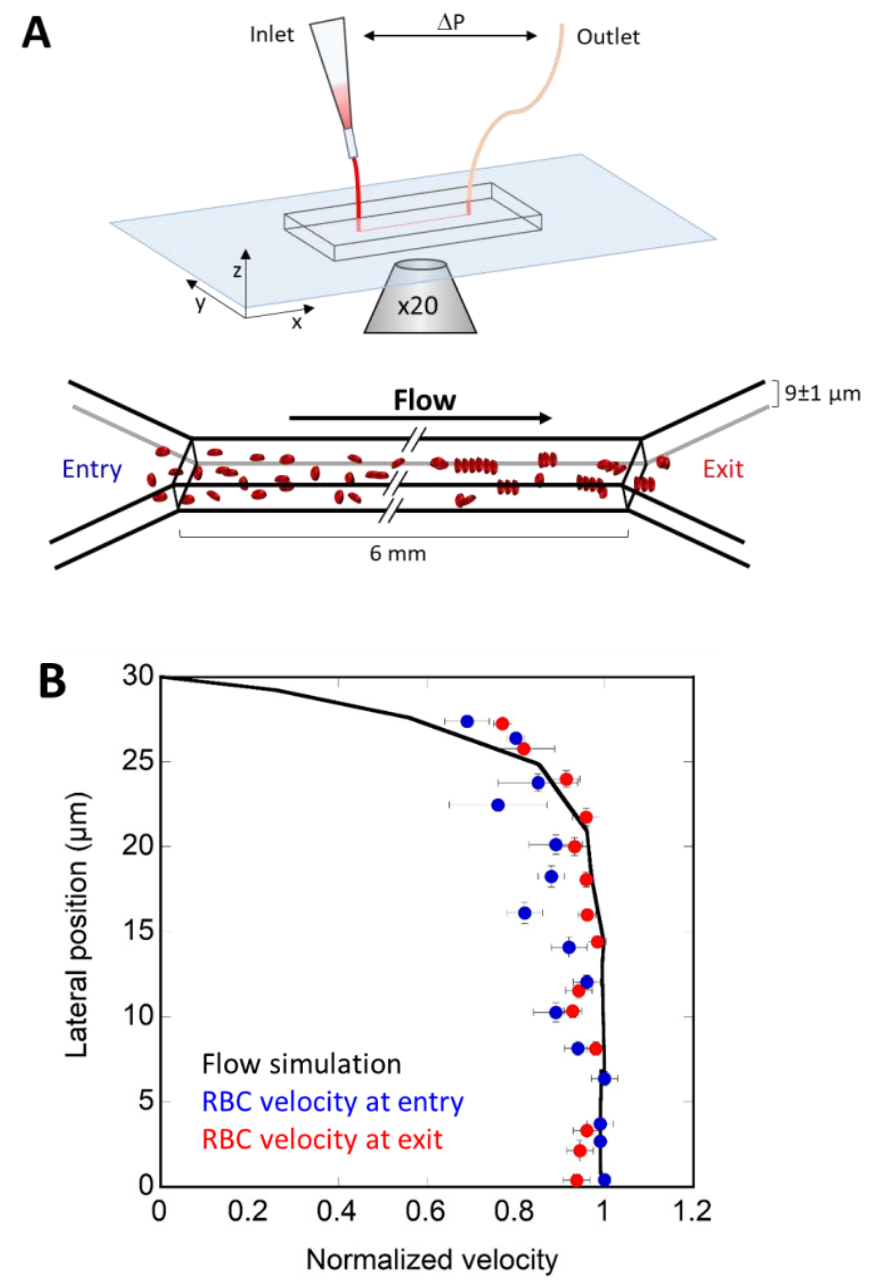

Figure 1 
A

$\stackrel{\bullet}{\Sigma}$

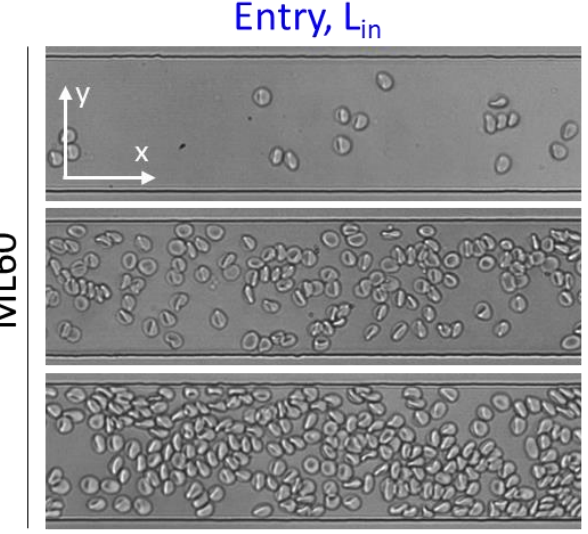

Exit, $\mathrm{L}_{\text {out }}$

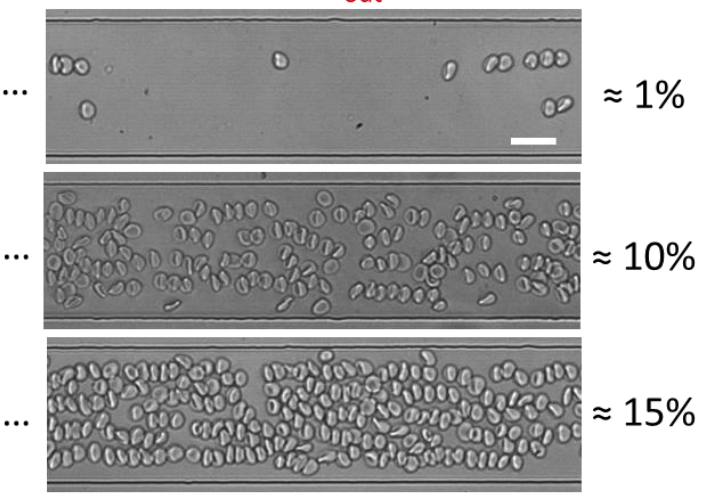

Exit, $\mathrm{L}_{\text {out }}$

B

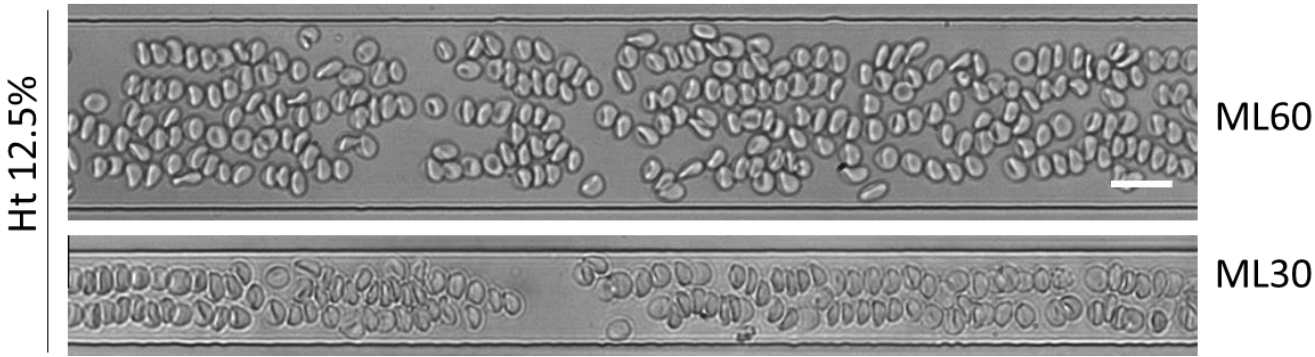

Figure 2. 


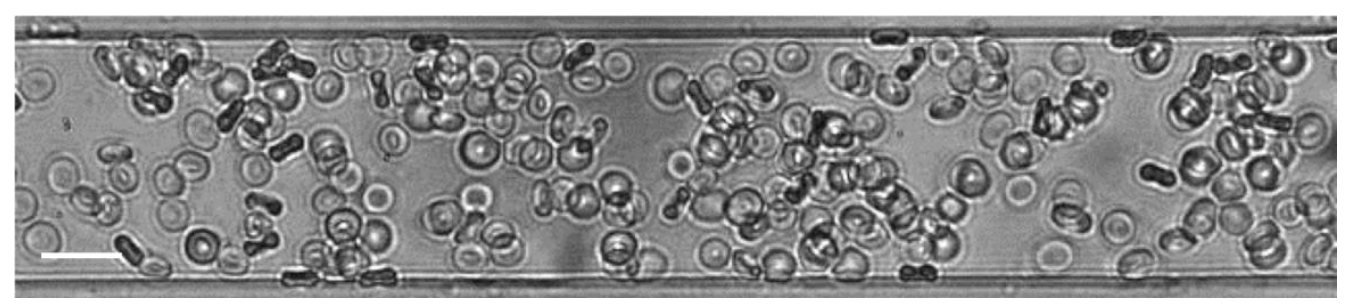

Figure 3. 

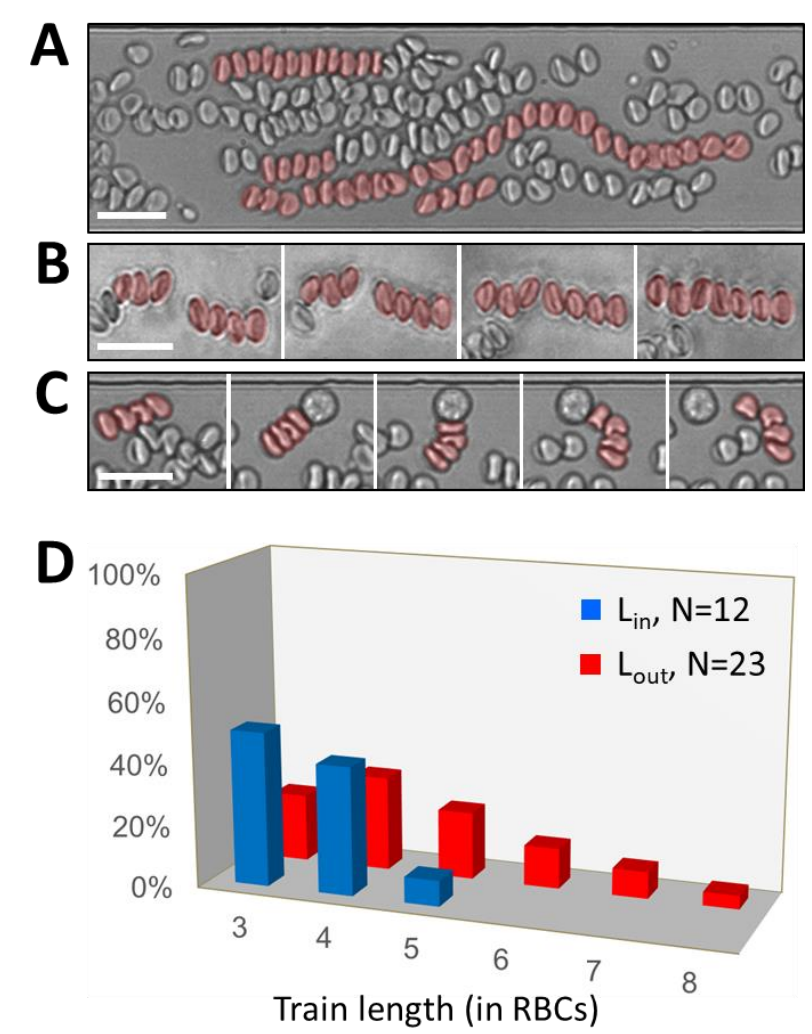

Figure 4. 
A
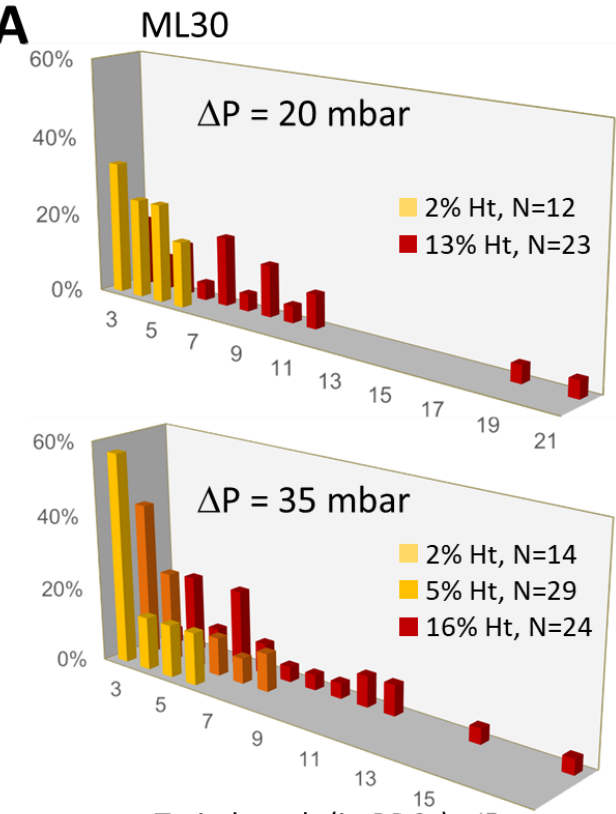

Train length (in RBCs)

\section{B}

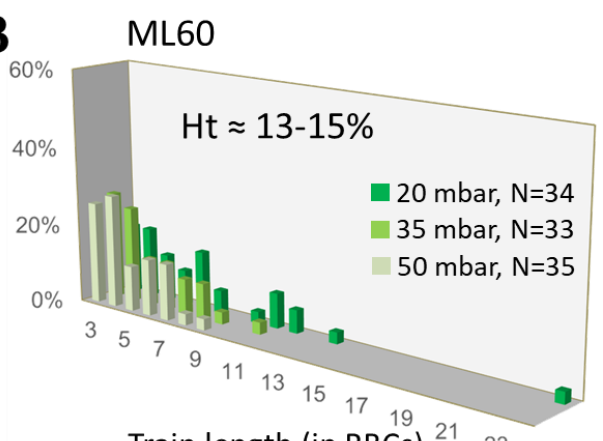

C

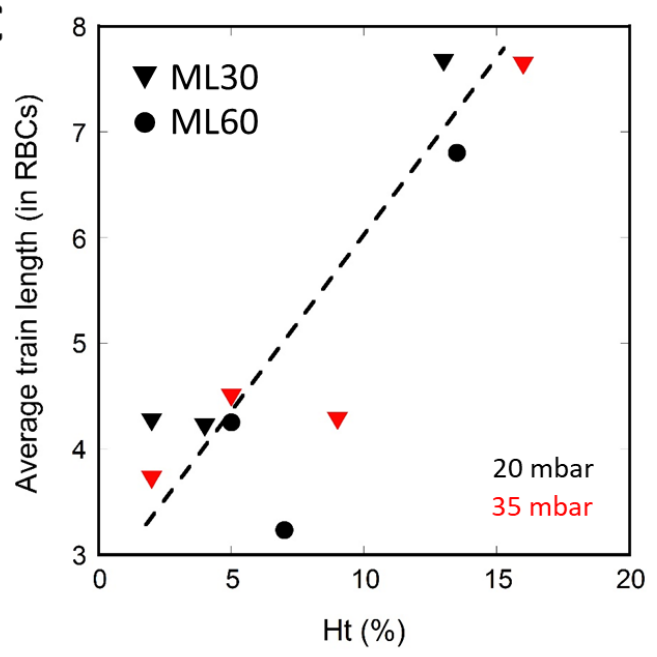

D

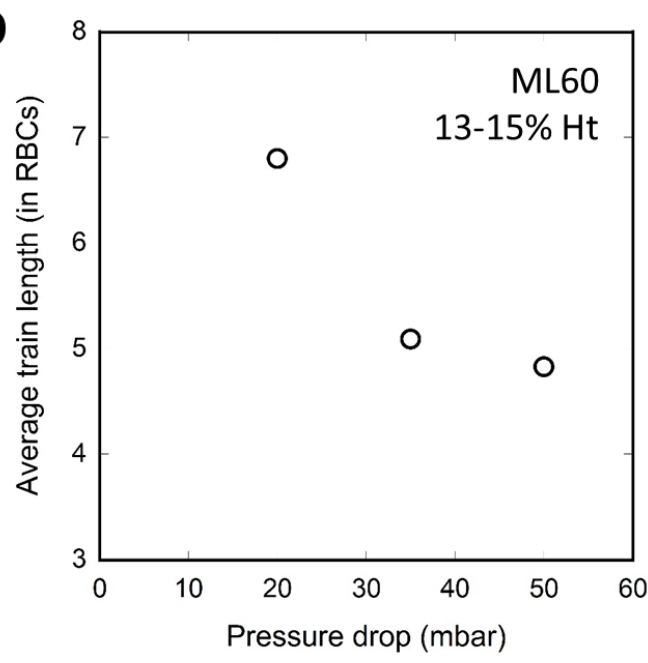

Figure 5. 
A
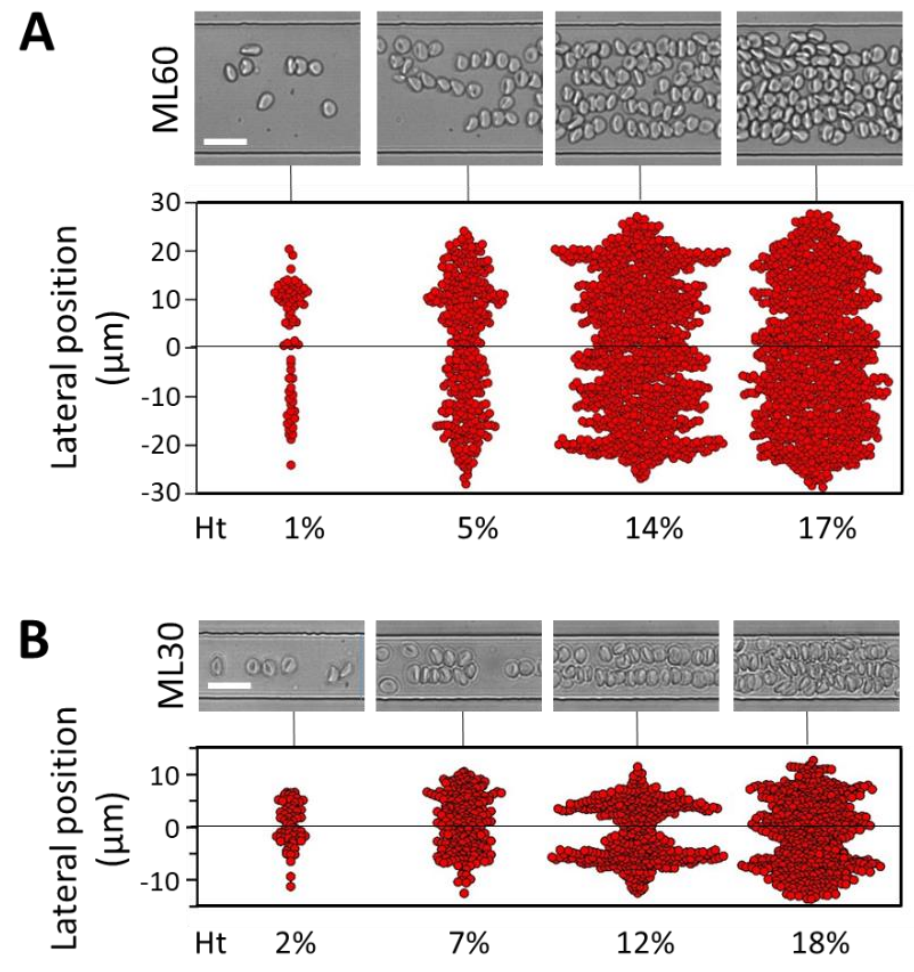

Figure 6. 

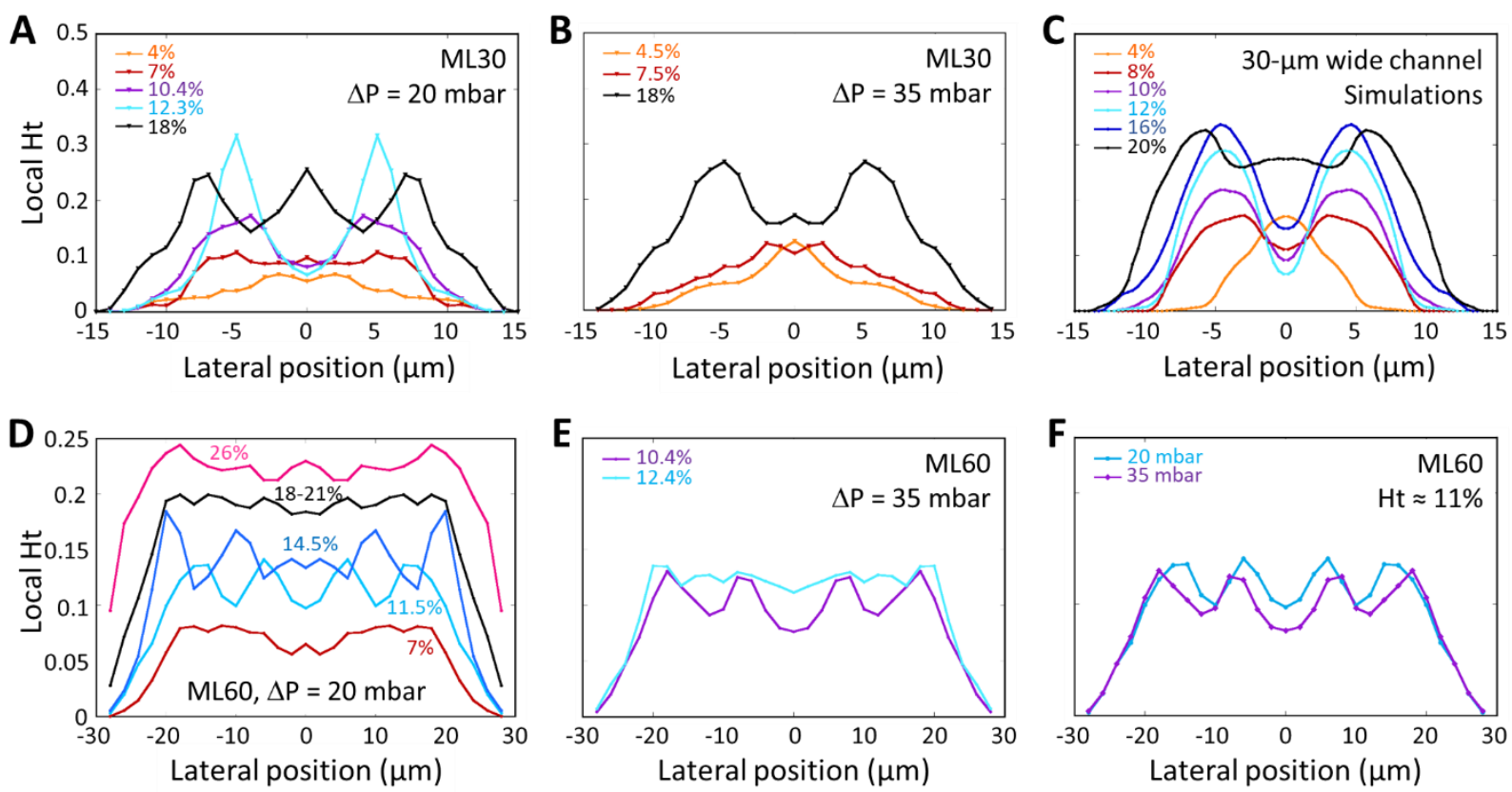

Figure 7. 

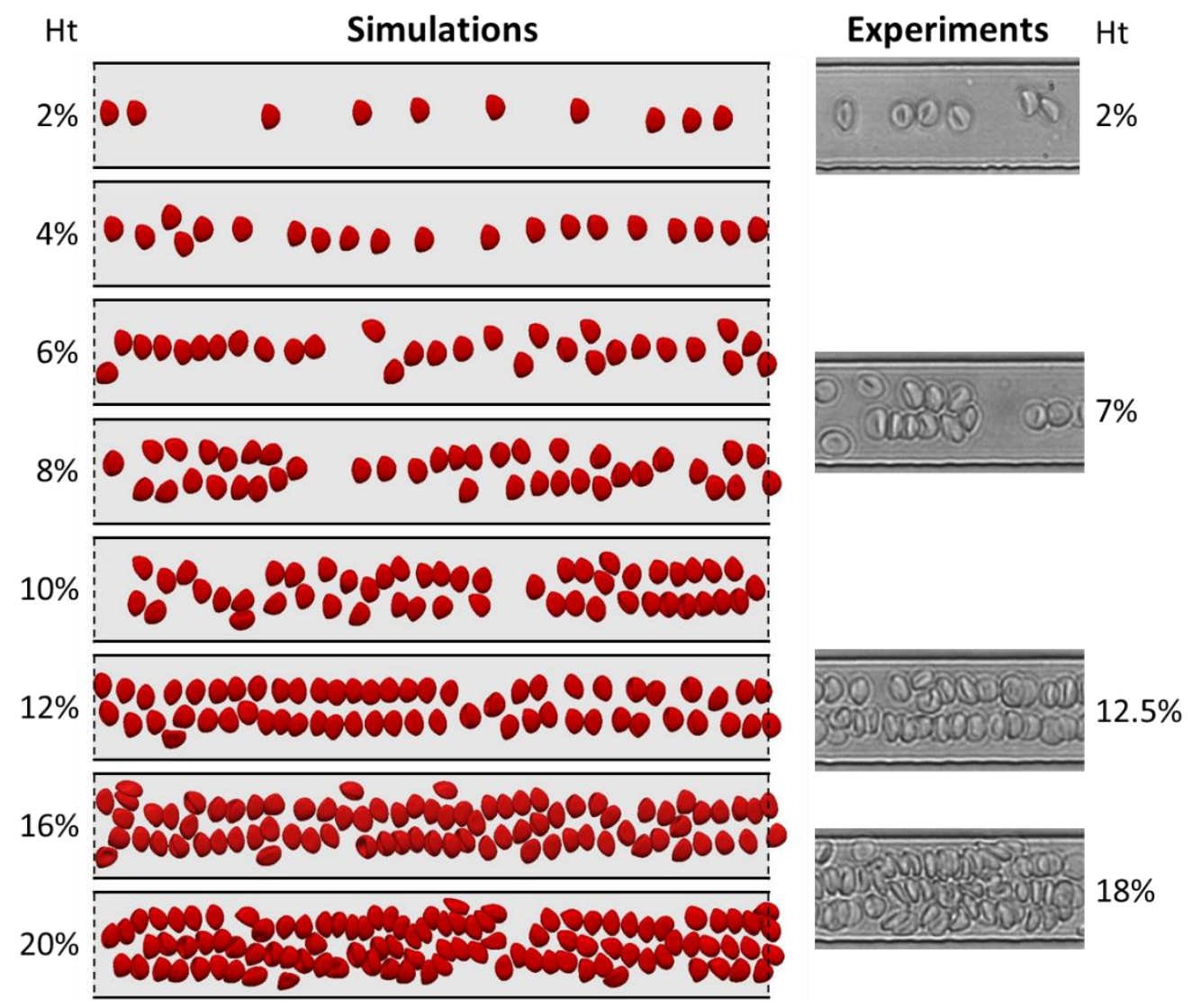

Figure 8. 


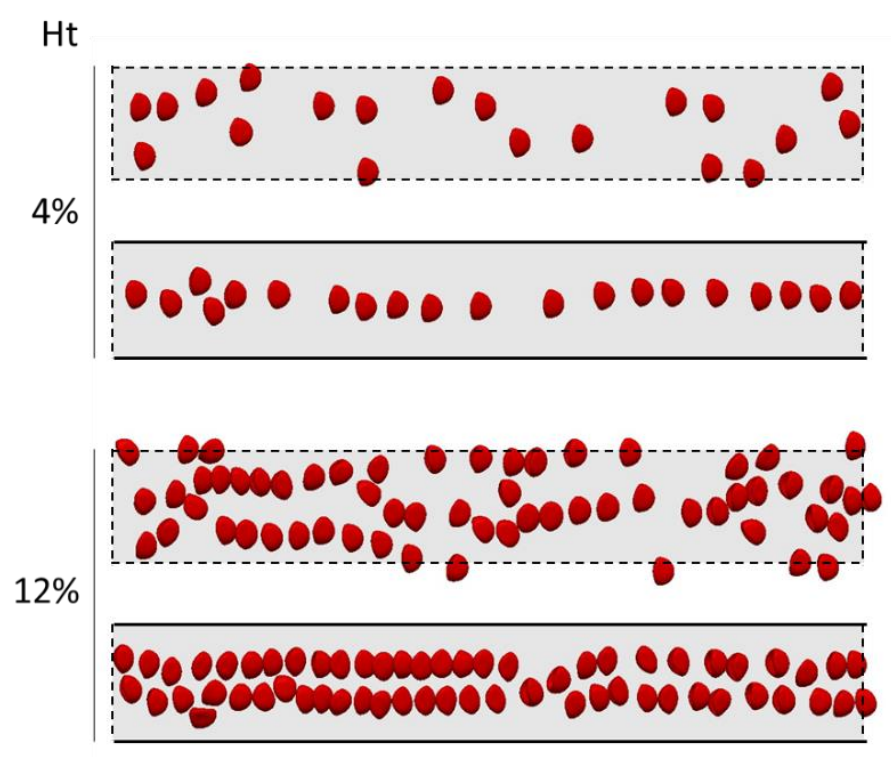

Figure 9. 
Capsule deformability

C1

$\mathrm{t}_{0}$

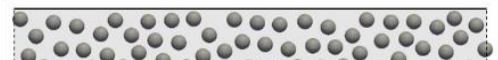

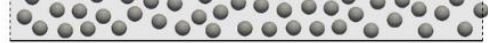
600 $8000.0 \%$

$t_{1}$ 8. 8088,0008

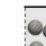

$t_{2}$ 00.080 .0000800

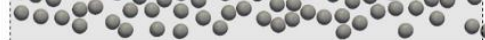

$t_{3}$

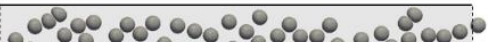

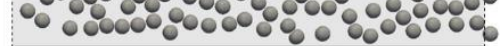
000008090098900 $t_{4}$
C2

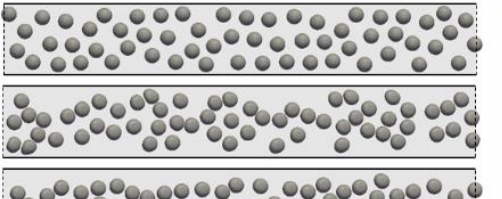

80080000000000000 - 000000800,000000

-

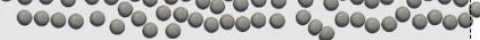

00000000000000000000 000000000000000000
C3

.0.0.0.0.000.0.000.0 0.000 .00000000 .000 0.

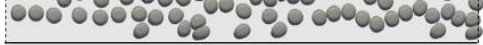

000.00 800008 0000000080 000008 00000

○०० 000000000000800 000 col-

-

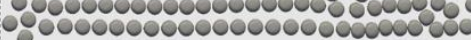

Figure 10. 


\section{Notes and references}

\section{Notes}

${ }^{\ddagger}$ http://imag.umontpellier.fr/ yales2bio/

\section{References}

${ }^{1}$ T. Vicsek and A. Zafeiris, Phys. Rep., 2012, 517, 71.

2 D. J. Sumpter, Collective Animal Behavior, Princeton University Press, 2010.

3 S. Camazine, Self-Organization in Biological Systems, Princeton University Press, 2003.

${ }^{4}$ D. Saintillan, Ann. Rev. Fluid Mech., 2017, 50, 563.

${ }^{5}$ G. Tomaiuolo, L. Lanotte, G. Ghigliotti, C. Misbah and S. Guido, Phys. Fluids, 2012, 24, 051903.

${ }^{6}$ V. Clavería, O. Aouane, M. Thiébaud, M. Abkarian, G. Coupier, C. Misbah, T. John and C. Wagner, Soft Matter, 2016, 39, 8235.

${ }^{7}$ P. Garstecki., M. J. Fuerstman, H. A. Stone and G. M. Whitesides, Lab Chip, 2006, 6, 437.

${ }^{8}$ C. Tufano, G. W. M. Peters and H. E. H. Meijer, Langmuir, 2008, 24, 4494.

9 A. Vananroye, P. Van Puyvelde and P. Moldenaers, Langmuir, 2006, 22, 2273 ; P. Van Puyvelde, A. Vananroye, R. Cardinaels and P. Moldenaers, Polymer, 2008, 49, 5363.

${ }^{10}$ Z. Shen, T. M. Fischer, A. Farutin, P. M. Vlahovska, J. Harting and C. Misbah, Phys. Rev. Lett., 2018, 120, 268102.

11 J.L. Mc Whirter, J. Liam, H. Noguchi and G. Gompper, Proc. Natl. Acad. Sci. U. S. A., 2009, 15, 6039-43.

${ }^{12}$ N. Takeishi and Y. Imai, Sci. Rep., 2017, 1, 5381.

13 T. Beatus, R. H. Bar-Ziv, and T. Tlusty, Phys. Rep., 2012, 516, 103.

14 P. J. A. Janssen, M. D. Baron, P. D. Anderson, J. Blawzdziewicz, M. Loewenbergd and E. Wajnryb, Soft Matter, 2012, 8, 7495.

${ }^{15}$ I. Shani, T. Beatus, R. H. Bar-Ziv and T. Tlusty, Nat. Phys., 2014, 10, 140.

${ }^{16}$ M. Abkarian, M. Faivre, R. Horton, K. Smistrup, C. A Best-Popescu and H. A Stone, Biomed. Mater., 2008,3, 034011.

${ }^{17}$ R. Fahraeus, Physiol. Rev., 1929, 9, 24.

${ }^{18}$ R. Fahraeus and T. Lindqvist, Am. J. Physiol., 1931, 96, 562.

${ }^{19}$ M. Abkarian and A. Viallat, Biophys. J., 2005, 89, 1055.

${ }^{20}$ P. Olla, Phys. Rev. Lett., 1999, 82, 453.

${ }^{21}$ T. W. Secomb, Ann. Rev. Fluid. Mech., 2017, 49, 443.

22 Y. C. Fung, Biomechanics, Springer, 1993.

${ }^{23}$ V. Moureau, P. Domingo and L. Vervisch , Comp. Rend. Mécan., 2011, 339, 141.

24 M. Malandain, N. Maheu and V. Moureau, J. Comp. Phys., 2013, 238, 32.

${ }^{25}$ S. Mendez, E. Gibaud and F. Nicoud, J. Comput. Phys., 2014, 256, 465.

26 J. Sigüenza, S. Mendez, D. Ambard, F. Dubois, F. Jourdan, R. Mozul and F. Nicoud, J. Comput. Phys., 2016, 322, 723. 
${ }^{27}$ M. Martins Afonso, S. Mendez and F. Nicoud, J. Fluid Mech., 2014, 746, 300.

28 J. Sigüenza, S. Mendez, and F. Nicoud, Biomech. Model. Mechanobiol., 2017, 16, 1645.

${ }^{29}$ L. Lanotte, J. Mauer, S. Mendez, D. A. Fedosov, J.M. Fromental, V. Clavería, F. Nicoud, G. Gompper and M. Abkarian, Proc. Natl. Acad. Sci. U. S. A., 2016, 113, 13289.

30 J. Mauer, S. Mendez, L. Lanotte, F. Nicoud, M. Abkarian, G. Gompper and D. A. Fedosov, Phys. Rev. Lett., 2018, 121, 118103.

${ }^{31}$ S. Mendez and M. Abkarian, Phys. Rev. Fluids, 2018, 3, 101101.

32 R. Skalak, A. Tozeren, R. P. Zarda, and S. Chien, Biophys. J., 1973, 13, 245-264.

${ }^{33}$ W. Helfrich, Z. Naturforsch. C, 1973, 28, 693.

${ }^{34}$ O. Y. Zhong-can and W. Helfrich, Phys. Rev. A, 1989, 39, 5280.

${ }^{35}$ P. Dimitrakopoulos, Phys. Rev. E, 2012, 85, 041917.

${ }^{36}$ S. Hénon, G. Lenormand, A. Richert and F. Gallet, Biophys. J., 1999, 76, 1145.

37 D. Cordasco, A. Yazdani, and P. Bagchi, Phys. Fluids, 2014, 26, 041902.

${ }^{38}$ Z. Peng, A. Mashayekh and Q. Zhu, J. Fluid Mech., 2014, 742, 96.

${ }^{39}$ G. H. W. Lim, M. Wortiz and R. Mukhopadhyay, Proc. Natl. Acad. Sci. U. S. A., 2002, 99, 16766.

40 J. Dupire, M. Abkarian and A. Viallat, Soft Matter, 2015, 11, 8372. 